\title{
Meibomian gland dysfunction: an overlooked eyelid disease
}

\begin{abstract}
Meibomian gland dysfunction is a multifactorial and chronic disease of the eyelids, leading to eye irritation, inflammation, evaporative and aqueous-deficient dry eye and negatively affecting the quality of life. MGD is often overlooked clinically. This review presents a general and practical guide for MGD diagnosis and management.
\end{abstract}

Keywords: Meibomian gland, dysfunction, dry eye, hypersecretory, hyposecretory, duct obstruction, lipid layer, tear film, ocular surface diseaset
Volume 8 Issue 3 - 2018

\author{
Burak Turgut,' Onur Çatak, ${ }^{2}$ Tamer Demir ${ }^{3}$ \\ 'Department of Ophthalmology, Yuksek Ihtisas University, Turkey \\ ${ }^{2}$ Department of Ophthalmology, Firat University, Turkey \\ ${ }^{3}$ Department of Ophthalmology, Onsekiz Mart University, \\ Turkey
}

Correspondence: Burak Turgut, Private Etimed Hospital, Ophthalmology Clinic, Elvan Mah. 1934. Sok. No:4 Etimesgut/ANKARA, Turkey, Tel +90 (3I2) 2930606 Email drburakturgut@gmail.com

Received: May 21, 2018 | Published: May 29, 2018

\section{Introduction}

Meibomian glands (MGs) are large sebaceous glands, vertically arranged in the tarsal plates of the upper and lower eyelids and produce the lipids of the outermost layer of the preocular tear film. The tarsal glands are firstly described by Heinrich MEIBOM (16381700), a professor of medicine at the university town of Helmsted, and afterward, these glands were called as MGs.

\section{Definition}

The term "Meibomian gland disease" is used to describe various disorders of MGs such as congenital lack, neoplastic and inflammatory disorders, replacement distichiasis and Meibomian gland dysfunction (MGD). The term has been first coined in the ophthalmic literature by Korb and Henriques in 1980s and it has been defined as a chronic MGD resulting in decreased secretion or poor quality of meibum. Lastly, MGD has been defined by subcommittee of The International Workshop on MGD (IWMGD) in 2011 as "'a chronic, diffuse abnormality of the MGs, commonly characterized by terminal duct obstruction and/or qualitative/quantitative changes in the glandular secretion which may result in alteration of the tear film, clinical apparent inflammation, OSD and symptoms of eye irritation". ${ }^{2-4}$

\section{The importance of the functions of MGs}

The functions of Meibomian lipids include providing a smooth optical surface for the cornea, reduction evaporation of the tear film during waking hours, lubrication during blinking, decreasing the surface tension, enhancing the stability and the spreading of the tear film, forming a barrier to prevent bacteria from entering the tear film, prevention of spillover of tears from the lid margin and the contamination of the tear film by sebum and sealing the opposing lid margins during sleep. ${ }^{2-9}$ MGD is one of the most common diseases of MGs. However, it is often overlooked clinically. MGD is a multifactorial and chronic disease of the eyelids, leading to eye irritation, inflammation and ocular surface disease (OSD). Additionally, it is well-documented that MGD is the leading cause of evaporative dry eye and also the most common underlying pathology in the cases with the aqueous-deficient dry eye. Additionally, MGD can negatively affect the quality of life..$^{2-9}$

\section{Risk factors}

The risk factors for MGD identified by the epidemiology and risk factors identification committee of International Meibomian Gland Dysfunction Study group are divided into three group as ophthalmic, systemic and therapeutic (Table 1). Ophthalmic risk factors include aniridia, chronic blepharitis (anterior or posterior), contact lens wearing, Demodex infestation of the eyelid, floppy lid syndrome, giant papillary conjunctivitis, ichthyosis, Salzmann nodular degeneration, and trachoma. In the other hand, systemic risk factors include especially in older age (increasing the amount of meibomian cells expressing estrogen receptors in the lower lid), ocular rosacea, hormonal disorders such as androgen insufficiency, Sjogren's syndrome, Stevens-Johnson syndrome, atopy, cicatricial pemphigoid and ectodermal dysplasia. The main therapeutic risk factors for MGD are reported as the usage of antiandrogen, antidepressant and antihistaminic drugs, retinoic acid for acne, drugs for benign prostatic hyperplasia and postmenopausal hormone therapeutics. ${ }^{10-13}$

\section{Symptoms}

The cases with MGD may be symptomatic or asymptomatic due to severe involvement of MGs. MGD is associated with various ocular symptoms may not only occur depending on the MGD itself but also on the secondary dry eye and ocular surface damage. The most common symptoms in the admission are fatigue, grittiness, dryness, burning or heavy sensation, stinging and itching in the eyes, redness and swelling on the eyelids and sometimes transient visual blurring. ${ }^{14-17}$ In case of suspected cases, detailed inquiries of the risk factors for MGD would be useful.

\section{Clinical findings and diagnosis}

MGD can present with thickening of the MGs secretions and eyelid margin, turbid secretions, plugged, deformed or cystoid and scarred 
MGs orifices, irregularity or deformation or a notch in the lower eyelid margin, anterior or posterior displacement of the mucocutaneous junction, telangiectatic/fine vessels crossing the MGs margins or orifices, vascular dilatation with or without inflammation, increased vascularity at the posterior border of the lid margin, madarosis or trichiasis, frothy or foamy tears, and bubbles in the tear film. ${ }^{2-8} \mathrm{~A}$ normal secretion from healthy MGs typically should seem clear and somewhat viscous similar to the olive or baby oil. A turbid or cloudy secretion may be an early sign of MGD while as plugged/obstructed/ scarred orifices and notch in the lower lid margin are the signs of advanced MGD. It is considered that the obstruction in orifices and terminal ducts of the MGs is identified as the most prominent aspect of MGD. On the ocular surface, evidence of dry eye and damage can be seen. Applying pressure with the finger on the region of MGs in the middle of the lower or upper eyelid may provide to reveal the absence of excretion of the meibum, to check the emptying of the MG content and to check the quality of the excretion. However, it is recommended the distinguishing evaporative dry eye from aqueous insufficiency due to MGD. For this purpose, initially, OSD indexing (OSDI) scoring should be performed. Then, the evaluation of blinking frequency and the time between two blink (blinking interval), measurements of tear film meniscus height on the lower eyelid conjunctiva, the tears osmolarity, fluorescein break up time or the ratio of break up time to blinking interval (Ocular protection index, $\mathrm{N}>1$ ), the evaluation of corneal staining with fluorescein and conjunctival staining with lissamine green and aqueous layer with Schirmer test. Following these evaluations revealed the dry eye, MGs functions, meibum excretion and quality with finger pressuring and grading of glandular morphology and loss with meibography should be evaluated..$^{2-9,14-17}$

In the dry eye-related tests, if the tears flow and volume are in the normal limits, an evaporative dry eye is considered, and the presence and severity of MGD can be determined by evaluating the MG morphology and functions. Meibography can reveal acinar atrophy and atrophic degeneration of the MGs. Additionally, non-contact meibography andOCT-meibography have provided important benefits in the diagnosis and follow-up of MGD. Meibometry, interferometry, evaporimetry, fluorophotometry, and meniscometry are other diagnostic tests which were used for etiological and detailed research in the diagnosis of MGD. Meibometry is a test to measure the amount of meibomian lipid at the edge of the steady state lid.Interferometry is a test allowing the visualization of lipid layer using optical principles. Based on the principle of elimination of the speed of disappearance of a dye applied to the ocular surface, fluorophotometry measures the production and volume of tears and the speed of regeneration. Evaporimetry shows the evaporation rate from the ocular surface. Meniscometry provides the objective measurement of height, radius, and volume of the tear film meniscus. The diagnosis of MGD is performed with evaluation of ocular symptoms, amount and quality of MG secretion, and lid margin abnormalities. ${ }^{2-9,14-17}$

\section{Stages}

International Working Group of Meibomian Gland Dysfunction has graded four stages MGD (Stage 1-Stage 4) to meibomian gland findings, ocular surface findings and the severity of the symptoms (Table 2). ${ }^{15}$

Classification and pathophysiology of MGD: The disturbed functions in MGD may be due to anatomic or secretory abnormalities of MGs and these leads to decreased tear film stability (evidenced by increased evaporation, increased surface tension, contamination with sebum, unsealed lid during sleep) and/or symptoms. MGD may be classified based on the severity, pathophysiologic or anatomic changes of the disease. In the last classification described by the International Meibomian Gland Dysfunction Task Group Identification and Classification Committee, MGD is divided into two groups according to the amount of secretion of the glands..$^{2-9,15}$

The low delivery group includes hyposecretory (Meibomian sicca) and obstructive-type $M G D$. Hyposecretory type is characterized by decreased lipid secretion without gland obstruction. Primer hyposecretion is usually associated with glandular atrophy. Obstructive MGD may occur in the case of a low secretion level. Histopathologic studies on obstructive MGD have shown squamous metaplasia and keratinized plugs at MG orifices, thickening of meibum and cellular debris within dilated ducts, MG hypertrophy, or atrophy. The reduction in the number of functional MGs is often associated with the wearing duration and use of contact lenses. Obstruction in MGs may be seen in older patients and patients who have previously used retinoid therapy for acne, and also the lack of androgen deficiency or androgen receptor. Obstructive MGD may occur as cicatricial or noncicatricial types. In cicatricial type, MG ducts and orifices displace mucosa backward while as, in non-cicatricial type, they locate at their normal anatomic positions. The main causes of cicatricial MGD are trachoma, ocular cicatricial pemphigoid, erythema multiforme, and atopic eye diseases. Noncicatricial MGD may occur in seborrheic dermatitis, acne rosacea, Sjogren's syndrome, atopy and psoriasis..$^{2-9,15}$ MG obstruction is the most common form of MGD. In initial stages of obstructive MGD, MGs continue to product meibum. However the meibum cannot excrete due to the obstruction in MG duct or orifices. This cause to increase intraglandular pressure within the MGs and consequently, the dilation of the duct and the acini, acinar degeneration and atrophy which may cause to the loss of meibocytes. Additionally, prolonged obstruction causes to bacterial colonization in MGs and the release of inflammatory mediators and lipolytic enzymes from bacteria. These lipolytic enzymes released by bacteria cause very irritating free fatty acids to breakdown the lipids in the tear film. Eventually, the loss of tear film integrity and stability results in an increased aqueous tear evaporation and evaporative dry eye. ${ }^{2-9,15}$

High delivery/secretory or seborrheic-type MGD (Meibomian seborrhea) is characterized by a high amount release of meibomian lipid at the lid margin when finger pressure applied on tarsae. It has main histopathologic changes including hypertrophy of the ductal epithelium and keratinization of the orifice epithelium. Hypersecretion of meibum into the tear film triggers an inflammatory reaction at the ocular surface. In primary hypersecretory type, there is no any associated disease. Secondary hypersecretory MGD can occur usually with seborrhoeic dermatitis, and sometimes in atopy and acne rosacea..$^{2-9,15}$

\section{Differential diagnosis}

The term "diffuse abnormality of the MGs used by IMGSG in the definition of MGD is critical. Because, in chalazia, a confused disease, the MGs are involved locally and, the involvement does not lead the tear film abnormalities or ocular surface epithelia. In the other hand, some inflammatory entities may be confused with MGD. However, the most common overlap in terminology is between MGD and posterior blepharitis. Meibomitis/meibomianitis is defined as subset of disorders with inflammation of the MG orifices. Anterior blepharitis is an inflammation of the lid margin anterior to the gray line and concentrated around the lashes. It may be accompanied by squamous 
debris or collarettes around the lashes, and inflammation may spill onto the posterior lid margin. Anterior blepharitis may simultaneously associate to MGD and in this case, concurrent treatment should be performed. Posterior blepharitis describes an inflammation of the posterior lid margin, which may have different causes, including MGD, conjunctival inflammation (allergic or infective), and/or other conditions, such as acne rosacea. Marginal blepharitis is inflammation in eyelid margin including both anterior and posterior blepharitis. ${ }^{2-}$ ${ }^{9,15}$ Meibomian keratoconjunctivitis has been defined as often associated with anterior blepharitis, with the most prominent changes centered on the meibomian glands. It is usually associated with some form of skin disease and is characterized by tear film instability, ocular surface inflammation, and ocular surface damage. It is an important cause of symptoms in severe chronic blepharitis. ${ }^{18,19}$ In MGD, inflammation is not essential; the term does not include neoplasia or congenital disease. The role of inflammation in the etiology of MGD is not clearly understood. However, associations between meibomian gland dropout and ocular surface inflammatory diseases, such as chronic blepharitis, giant papillary conjunctivitis, and Sjogren syndrome, histopathologic lipogranulomatous inflammation around the gland lobules and increased vascularization of the posterior lid margin support to be have a role of inflammation in pathophysiology. ${ }^{2-9}$

\section{Management}

The management of MGD is performed based on MGD staging according to the severity of the disease and possible accompanying diseases (Table 2) ${ }^{20-22}$ The main purpose of the management should improve the symptoms and quality of life of the patient. The cases in Stage 1 should be informed about the chronic nature of the disease and the lifestyle changes, the potential impact of diet and the effect of work/ home environments on tear evaporation, and the possible drying effect of certain systemic medications. In this stage, eyelid hygiene including warming/expression/baby shampoo or eyelid wipes and removing the obstructed meibum may be considered. ${ }^{20-22}$ In the patients with Stage 2 MGD, advisement on improving ambient humidity; optimizing workstations and increasing dietary omega-3 fatty acid intake, eyelid hygiene with eyelid warming (a minimum of four minutes, once or twice daily) followed by moderate to firm massage and expression of MG secretions. Additionally, artificial lubricants preservative-free, topical emollient lubricant or liposomal spray and the use of topical azithromycin should be prescribed and oral tetracycline derivatives should be considered. ${ }^{20-22}$ In Stage 3, addition to recommendations and medications, oral tetracycline or minocycline or doxycycline, lubricant ointment at bedtime and anti-inflammatory therapy for dry eye in indicated cases should be advised. ${ }^{20-22}$ The patients with Stage 4 MGD may be presacribed topical anti-inflammatory drugs. Topical anti-inflammatory preparations include cyclosporine or corticosteroid are very effective in reducing inflammation in patients with MGD. ${ }^{20-22}$

Plus disease is the stage which diseases to the eyelid and ocular surfaces (causative or secondary to MGD or incidental) such as other exacerbated inflammatory OSD, mucosal keratinization, phlyctenular keratitis, trichiasis, cicatricial conjunctivitis, ocular cicatricial pemphigoid, chalazion, anterior blepharitis and Demodex-related anterior blepharitis with cylindrical dandruff associated to MGD. Simultaneously treatment modality to stage and specific treatment of these diseases should be considered with the use of pulsed soft steroid, bandage contact lens/scleral contact lens, topical N-acetylcysteine and cyclosporine A, steroid therapy, epilation, cryotherapy, intralesional steroid or excision, topical antibiotic or antibiotic/steroid and Tea tree oil scrubs (specifically the terpenin-4-ol component) (Table 2). ${ }^{20-22}$ Except for above-mentioned treatment modalities, in selected cases, other manual and mechanical but invasive techniques such as lid margin debridement, intraductal meibomian gland probing, MG probing, Intense Pulsed Light and LipiFlow ${ }^{\circledR}$ thermal pulsation system may be tried. ${ }^{20-24}$

Table I Risk factors for MGD

\begin{tabular}{ll}
\hline Risk factors for MGD \\
\hline Ocular & Aniridia \\
& Chronic blepharitis (anterior or posterior) \\
& Contact lens wearing \\
& Demodex infestation of eyelid \\
& Floppy lid syndrome \\
& Giant papillary conjunctivitis \\
& Ichthyosis \\
& Salzmann nodular degeneration \\
& Trachoma \\
Systemic & Older age \\
& Rosacea \\
& Androgen insufficiency \\
& Sjogren's syndrome \\
& Stevens-Johnson syndrome \\
& Atopy \\
& Cicatricial pemphigoid \\
& Ectodermal dysplasia \\
& Antiandrogens \\
& Antidepressants \\
& Antihistaminics \\
& Retinoic acid \\
Therapeutic & Drugs for benign prostatic hyperplasia \\
& Postmenopausal hormone therapeutics \\
\hline
\end{tabular}


Table 2 A brief guide for MGD diagnosis and treatment

NOTE:Adapted from the relevant table in "Report of the Subcommittee on Management and Treatment of Meibomian Gland Dysfunction" of The International Workshop on Meibomian Gland Dysfunction ${ }^{19}$

Source: https://doiorg/ I0 I I67/iovs I0-6997g

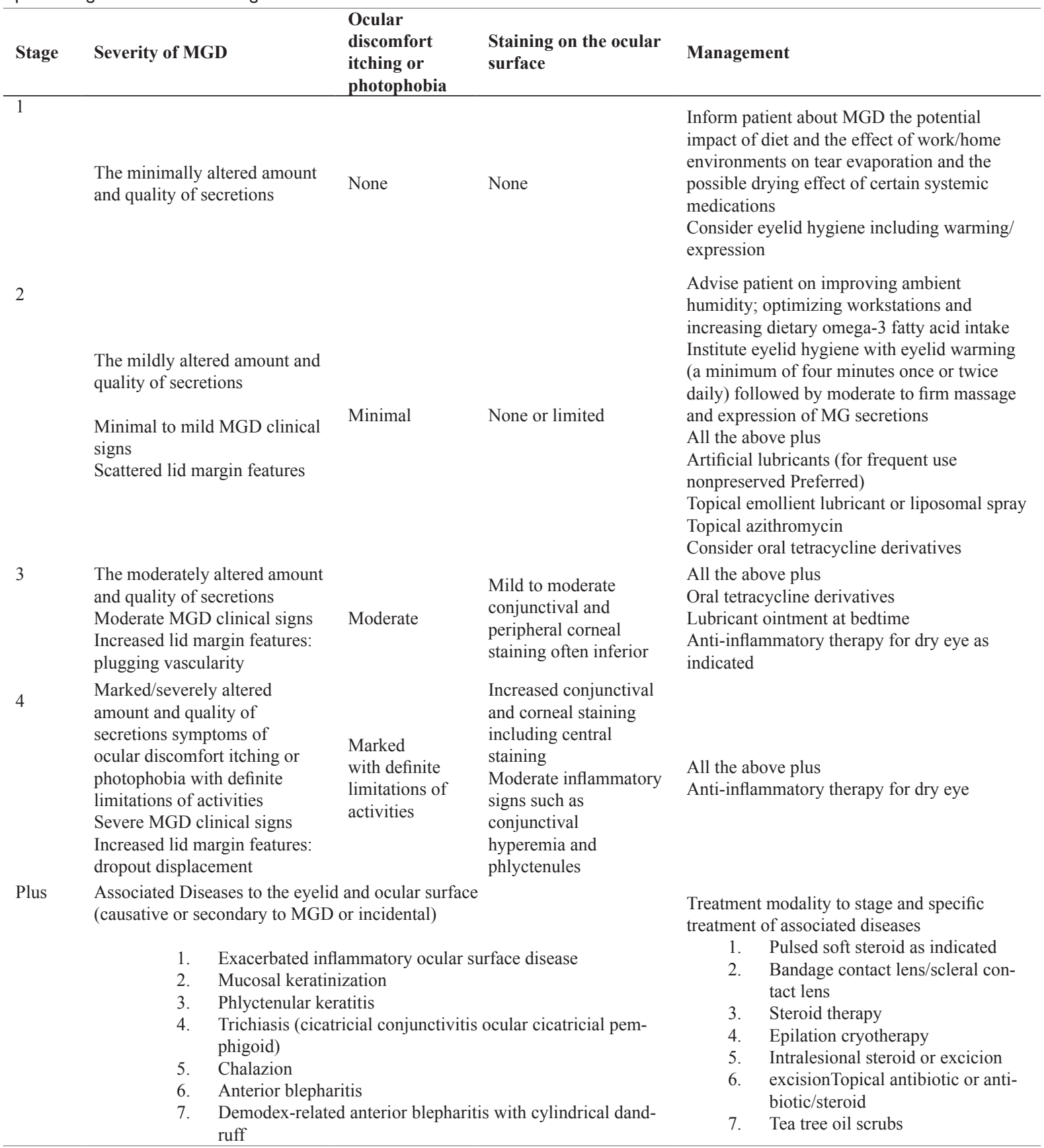

\section{Acknowledgments}

None.

\section{Conflict of interest}

The authors declare that there is no conflict of interest regarding the publication of this paper.

\section{Funding}

The authors received no financial support for the research, authorship, and/or publication of this article.

\section{Authorship contributions}

Concept, design and data collection: Burak Turgut; literature Search: Onur Çatak, Tamer Demir; writing, analysis, interpretation: 
Burak Turgut, Onur Çatak, Tamer Demir.

\section{References}

1. Korb DR, Henriquez AS. Meibomian gland dysfunction and contact lens intolerance. J Am Optom Assoc. 1980;51(3):243-251.

2. Foulks GN, Bron AJ. Meibomian gland dysfunction: a clinical scheme for description, diagnosis, classification, and grading. Ocul Surf. 2003;1(3):107-126.

3. Opitz DL, Harthan JS, Fromstein SR, et al. Diagnosis and management of meibomian gland dysfunction: optometrists' perspective. Clinical Optometry 2015;7:59-69.

4. Knop E, Knop N, Millar T, et al. The International Workshop on Meibomian Gland Dysfunction: Report of the subcommittee on anatomy, physiology, and pathophysiology of the meibomian gland. Invest Ophthalmol Vis Sci. 2011;52(4):1938-1978.

5. Bron AJ, Benjamin L, Snibson GR. Meibomian gland disease. Classification and grading of lid changes. Eye (Lond). 1991;5(Pt 4):395-411.

6. Chhadva P, Goldhardt R, Galor A. Meibomian Gland Disease: The Role of Gland Dysfunction in Dry Eye Disease. Ophthalmology. 2017;124(11S):S20-S26.

7. Arita R, Fukuoka S, Morishige N. New insights into the morphology and function of meibomian glands. Exp Eye Res. 2017;163:64-71.

8. Shimazaki J, Sakata M, Tsubota K. Ocular surface changes and discomfort in patients with meibomian gland dysfunction. Arch Ophthalmol. 1995;113(10):1266-1270.

9. Suzuki T, Tomo S, Kinoshita S. Meibomian Glands and Ocular Surface Inflammation. Ocular Surf. 2015;13(2):133-149.

10. Sridharan K, Sivaramakrishnan G. Therapies for Meibomian Gland Dysfunction: A Systematic Review and Meta-Analysis of Randomized Controlled Trials. Open Ophthalmol J. 2017;11:346-354.

11. Machalińska A, Zakrzewska A, Safranow K, et al. Risk Factors and Symptoms of Meibomian Gland Loss in a Healthy Population. $J$ Ophthalmol. 2016;2016:7526120.

12. Arita R, Fukuoka S, Morishige N. Meibomian Gland Dysfunction and Contact Lens Discomfort. Eye Contact Lens. 2017;43(1):17-22.
13. Viso E, Millán AC, Rodríguez-Ares MT. Rosacea-associated Meibomian Gland Dysfunction - An Epidemiological Perspective. European Ophthalmic Review 2014;8(1):13-16.

14. Wise RJ, Sobel RK, Allen RC. Meibography: A review of techniques and technologies. Saudi J Ophthalmol. 2012;26(4):349-356.

15. Nelson JD, Shimazaki J, Benítez del Castillo JM, et al. The international workshop on meibomian gland dysfunction: report of the definition and classification subcommittee. Invest Ophthalmol Vis Sci. 2011;52(4):1930-1937.

16. Geerling G, Baudouin C, Aragona P. Emerging strategies for the diagnosis and treatment of meibomian gland dysfunction: Proceedings of the OCEAN group meeting. Ocul Surf. 2017;15(2):179-192.

17. Milner MS, Beckman KA, Luchs JI, et al. Dysfunctional tear syndrome: dry eye disease and associated tear film disorders - new strategies for diagnosis and treatment. Curr Opin Ophthalmol. 2017;28(Suppl 1):347.

18. Bron AJ, Tiffany JM. The contribution of meibomian disease to dry eye. Ocul Surf. 2004;2(2):149-165.

19. McCulley JP, Sciallis GF. Meibomian keratoconjunctivitis. Am J Ophthalmol. 1977;84(6):788-793.

20. Geerling G, Tauber J, Baudouin C, et al. The International Workshop on Meibomian Gland Dysfunction: Report of the Subcommittee on Management and Treatment of Meibomian Gland Dysfunction. Invest Ophthalmol Vis Sci. 2011;52(4):2050-2064.

21. Qiao J, Yan X. Emerging treatment options for meibomian gland dysfunction. Clin Ophthalmol. 2013;7:1797-1803.

22. Thode AR, Latkany RA. Current and Emerging Therapeutic Strategies for the Treatment of Meibomian Gland Dysfunction (MGD). Drugs. 2015;75(11):1177-1185.

23. Lane SS, DuBiner HB, Epstein RJ, et al. A new system, the LipiFlow, for the treatment of meibomian gland dysfunction. Cornea. 2012;31(4):396-404.

24. Craig JP, Chen YH, Turnbull PR. Prospective trial of intense pulsed light for the treatment of meibomian gland function. Invest Ophthalmol Vis Sci. 2015;56(3):1965-1970. 